\title{
Improvement in Breast Lesion Characterization With Dynamic Contrast-Enhanced MRI Using Pharmacokinetic Modeling and Bookend $T_{1}$ Measurements
}

\author{
Greg O. Cron, ${ }^{1 *}$ Frederick Kelcz, ${ }^{2}$ and Giles E. Santyr ${ }^{1}$
}

\begin{abstract}
Dynamic contrast-enhanced breast MR imaging was performed on 14 patients (five cancerous lesions, nine benign) with sliceselective spoiled gradient-recalled echo (2D SPGR) imaging. Adiabatic saturation recovery $T_{1}$ measurements were performed before ( $\left.T_{1 \text { pre }}\right)$ and after $\left(T_{1 \text { post }}\right)$ 2D SPGR imaging. These two "bookend" $T_{1}$ measurements were used to calibrate the equations which were employed to convert the time course of the 2D SPGR signal strength to $T_{1}$-vs.-time, which in turn was used to compute the gadolinium concentration-vs.-time $([\mathrm{C}](\mathrm{t}))$ in the lesion. The extraction-flow product (EF) was computed for each lesion by pharmacokinetic modeling of $[\mathrm{C}](\mathrm{t})$. For this study, EF provided a sensitivity and specificity for cancer of $100 \%$ and $78 \%$, respectively. When only $T_{1 \text { pre }}$ was used to estimate [C](t) (which assumes a priori knowledge of the shape and amplitude of the slice profile), the sensitivity and specificity fell to $80 \%$ and $56 \%$, respectively. This is presumably due to unexpected variations in the shape and/or amplitude of the slice profile, which could be caused by factors such as patient-to-patient variations in breast geometry or inconsistently set transmit gains. Therefore, both $T_{1 \text { pre }}$ and $T_{1 \text { post }}$ measurements are necessary for optimum sensitivity and specificity using pharmacokinetic analysis. Magn Reson Med 51: 1066-1070, 2004. (c) 2004 Wiley-Liss, Inc.
\end{abstract}

Key words: magnetic resonance imaging (MRI); breast cancer; gadolinium contrast agent

Quantitative $T_{1}$-weighted dynamic contrast-enhanced MRI $\left(T_{1}\right.$-w DCE-MRI) is used by many researchers for detecting and evaluating breast disease (1-6). This technique involves intravenous injection of a gadolinium (Gd) contrast agent during rapid ( $\sim$ sec), repeated $T_{1}$-weighted imaging. From these images, the Gd concentration-vs.-time $([\mathrm{C}](\mathrm{t}))$ of a lesion of interest can be estimated (1-4,6,7). Pharmacokinetic modeling can then be applied to $[\mathrm{C}](\mathrm{t})$ to extract parameters such as the extraction-flow product (EF, which is equivalent to $\left.\mathrm{K}^{\text {trans}}\right)(1,4,5,8)$. These parameters are useful for lesion diagnosis and tracking of treatment progress.

A broad class of pulse sequences frequently used for $T_{1}$-w DCE-MRI is slice-selective (i.e., two-dimensional) spoiled gradient-recalled echo (2D SPGR) imaging $(2,3,6,7,9,10)$. Such pulse sequences are often used for

\footnotetext{
${ }^{1}$ Department of Physics, Carleton University, Ottawa, Ontario, Canada.

${ }^{2}$ Department of Radiology, University of Wisconsin, Madison, Wisconsin.

This work was presented in part at the Eleventh Meeting of the ISMRM, Toronto, 2003.

Grant sponsor: U.S. National Cancer Institute; Grant number: R29 CA5984005; Grant sponsor: Canadian Breast Cancer Research Initiative.

*Present address and correspondence to: Greg O. Cron, c/o Prof. Bernard Gallez, Lab. of Biomed. MR, Catholic Univ. of Louvain, CMFA/REMA, Avenue Mounier 73.40, B-1200 Brussels, Belgium.

E-mail: Gregory.Cron@rema.ucl.ac.be

Received 21 February 2003; revised 1 December 2003; accepted 1 December 2003.

DOI 10.1002/mrm.20042

Published online in Wiley InterScience (www.interscience.wiley.com).

(C) 2004 Wiley-Liss, Inc.
}

$T_{1}$-W DCE-MRI because they provide relatively artifact-free images with the high temporal resolution $(<\sim 15 \mathrm{sec})$, which is useful for differentiating benign from malignant breast lesions (11).

The signal-vs.-time (S(t)) of a pixel or region of interest (ROI) in a lesion can be measured directly from the 2D SPGR images. The goal is then to convert $\mathrm{S}(\mathrm{t})$ to $T_{1}(\mathrm{t})$, which can subsequently be used to compute [C](t) for pharmacokinetic modeling $(1,4,9,12)$. The $T_{1}(t)$ can be estimated from $\mathrm{S}(\mathrm{t})$ by utilizing a 2D SPGR signal strength equation which gives the relationship between $S$ and $T_{1}$. The signal strength equation has an overall scaling factor which must be determined either by measuring $T_{1}$ before injection of the $\mathrm{Gd}$ contrast agent $\left(T_{1 \text { pre }}\right)$ or by measuring one or more values of $S$ obtained with special flip angles and TR settings $(4,6,7,9,13)$.

Unfortunately, the 2D SPGR signal strength is very sensitive to even modest variations in the transmit magnetic field $\left(B_{1}\right)$, which can cause the signal strength equation to become more and more inaccurate as the $T_{1}$ shortens (and [C] increases) postinjection (7). This can lead to significant errors $(>50 \%)$ in the estimated $[\mathrm{C}](\mathrm{t})(7)$. Inaccuracies in $[\mathrm{C}](\mathrm{t})$ are undesirable because they lead to errors in the estimated pharmacokinetic parameters, reducing sensitivity and specificity and making the method less consistent for tracking treatment progress.

We have demonstrated that a second measurement of $T_{1}$ (denoted $T_{1 \text { post }}$ ), performed after the dynamic contrastenhanced 2D SPGR imaging, can minimize the errors in the estimated $T_{1}(\mathrm{t})$ (7). This second $T_{1}$ measurement is an important part of the "Bookend Method" that we have developed, which allows $T_{1}(\mathrm{t})$ to be estimated accurately $(<\sim 10 \%$ error $)$ from $2 \mathrm{D}$ SPGR image data, even in the presence of variations in $B_{1}$ or the slice-select profile shape (7). For the Bookend Method, $T_{1 \text { pre }}$ and $T_{1 \text { post }}$ are employed to calibrate and correct the 2D SPGR signal strength equation used to estimate $T_{1}(\mathrm{t})$.

The purpose of this work was to validate the clinical usefulness of the Bookend Method for quantitative $T_{1}-\mathrm{W}$ DCE-MRI of the breast. In order to do this, it was first necessary to develop a reasonably fast $(\sim \min )$ clinical $T_{1}$ measurement imaging pulse sequence which would be insensitive to variations in $B_{1}$. The Bookend Method was then performed on a group of 14 patients (five cancerous lesions, nine benign lesions). For each lesion, EF was calculated from the estimated $[\mathrm{C}](\mathrm{t})$ data and compared to pathological diagnosis. In order to compare the Bookend Method with a more conventional approach of estimating $[\mathrm{C}](\mathrm{t})$, the entire analysis was repeated using $T_{1 \text { pre }}$ but ignoring $T_{\text {1post }}$. 


\section{MATERIALS AND METHODS}

This study was approved by the Kingston General Hospital and Ottawa Hospital Human Research Ethics Boards. Fourteen patients with either palpable lumps or mammographically or sonographically suspicious lesions were recruited who were scheduled for surgical biopsy. Informed written consent was obtained from each patient prior to each MR imaging exam. Imaging was performed with $1.5 \mathrm{~T}$ Signa Imaging Systems (General Electric, Milwaukee, WI) and a transmit/receive breast coil (MRI Devices, Milwaukee, WI).

For each patient, the lesion was first localized with conventional MRI pulse sequences (20). Subsequently, the following protocol was used:

1. ( $T_{1 \text { pre }}$ measurement) adiabatic saturation recovery (ASR) 8 slices, recovery times $(\tau)=230,635,1329$, then $2920 \mathrm{~ms}$. This $T_{1}$ measurement technique is described in more detail below. The total imaging time for this step was $\sim 10 \mathrm{~min}$. For this and subsequent pulse sequences, coronal 5-mm-thick contiguous slices, $256 \times 128$ matrix size, and $16 \mathrm{~cm} \mathrm{FOV}$ were used.

2. (2D SPGR imaging) $T_{1}$-weighted dynamic contrastenhanced fast spoiled gradient echo (2D FSPGR) $\mathrm{TR}=10 \mathrm{~ms}$, TE $=3 \mathrm{~ms}$, flip angle $=20^{\circ}, 2 \mathrm{NEX}, 5-8$ slices, temporal resolution $=\sim 12-19$ sec, $0.1 \mathrm{mmol} / \mathrm{kg}$ Omniscan (Nycomed, Brampton ON, Canada) injected as a bolus after the fourth complete image set (i.e., fourth time point), total duration of dynamic imaging $=\sim 10 \mathrm{~min}$.

3. ( $T_{1 \text { post }}$ measurement) Same as 1 except that the recovery times $(\tau)$ were $230,432,775$, then $2920 \mathrm{~ms}$.

Postprocessing was performed off-line with Matlab (MathWorks, Natick MA). For each 2D SPGR imaging slice which contained part of the lesion, an ROI was manually drawn on the area of the lesion which demonstrated the greatest signal enhancement, defined as $\Delta \mathrm{S}=\mathrm{S}(\mathrm{t}=2 \mathrm{~min})-$ $\mathrm{S}(\mathrm{t}=0 \mathrm{~min})$. Each ROI covered a group of contiguous pixels for which $\Delta S$ was at least $80 \%$ of the maximum $\Delta S$ for that slice.

Each ROI was propagated through the saturation recovery and 2D SPGR images. $T_{1 \text { pre }}$ and $T_{1 \text { post }}$ were each determined with a three-parameter fit to the four recovery points. For each ROI, $T_{1}(\mathrm{t})$ was estimated from $\mathrm{S}(\mathrm{t})$ using the Bookend Method analysis (Method 4 in Ref. 7). For this analysis, $T_{1 \text { pre }}$ and $T_{1 \text { post }}$ were used to correct a lookup table that relates $S$ and $T_{1}(7)$. The change in $1 / T_{1}$ as a function of time was converted to $[\mathrm{C}](\mathrm{t})$ by dividing by $4.5 \mathrm{mM}^{-1} \mathrm{~s}^{-1}(12)$. The conversion of $T_{1}(\mathrm{t})$ to $[\mathrm{C}](\mathrm{t})$ assumed that the intravascular component was insignificant and that there was slow exchange of water between the plasma and the extravascular space (14). Under these assumptions, $[\mathrm{C}](\mathrm{t})$ represents the time course of the average concentration of Gd in the extravascular space (14).

The $[\mathrm{C}](\mathrm{t})$ data were fit to a well-known pharmacokinetic model:

$$
\frac{d[C](t)}{d t}=E F\left[C_{a}(t)-\frac{[C](t)}{v}\right] .
$$

For this equation, $\mathrm{C}_{\mathrm{a}}(\mathrm{t})$ is the arterial input function (AIF), defined as the concentration-vs.-time of $\mathrm{Gd}$ in the plasma of the arterial blood supply. For this study, $\mathrm{C}_{\mathrm{a}}(\mathrm{t})$ was not measured for each patient. Instead, an approximate average of published data was used $(15,16)$. The peak amplitude $(2.7 \mathrm{mM})$ of the initial bolus phase $(t=0$ to $\sim 25 \mathrm{~s})$ of this average AIF was calculated by averaging the peak amplitudes of the six AIF measurements shown in fig. 8 of Fritz-Hansen et al. (15). The initial, rapid washout of the average AIF from that peak value ( $t=\sim 25$ to $50 \mathrm{sec}$ ) was modeled as a simple monoexponential decay with no recirculation. The rate constant for this decay $\left(0.12 \mathrm{~s}^{-1}\right)$ was calculated by fitting a monoexponential decay to the initial washout phase of each of the Fritz-Hansen curves, then averaging the resultant rate constants. The longer, slower washout phase of the average AIF ( $t=\sim 50-600 \mathrm{sec}$ ) was calculated similarly from data published by Weinmann et al. (16).

The unitless quantity $v$, a free-floating parameter for the curve fit of Eq. 1, is the volume fraction of extravascular space occupied by the extracellular interstitial space (i.e., the space outside the cells and capillaries). The term EF (the other free-floating parameter) is known as the extraction-flow product. EF was expressed in units of $\mathrm{ml} /(\mathrm{min}$ $100 \mathrm{~g}$ ), under the assumption of unit density of tissue, to facilitate comparison to other studies $(4,5,8)$. To further facilitate such comparison, the value of EF extracted from the curve fit was subsequently divided by one minus the hematocrit ( $\mathrm{Hc}=40 \%$ assumed). This adjustment made the EF values more consistent with those of many other investigators who use whole blood concentrations for $\mathrm{C}_{\mathrm{a}}(\mathrm{t})$ instead of plasma concentrations (4).

For each lesion, a set of EF values was therefore obtained which corresponded to the set of ROIs which had been drawn. The maximum EF value in that set was defined as the one which was representative of the lesion. As a comparison, the entire analysis was repeated using the ROI which corresponded to that maximum EF value, this time assuming that there were no imperfections in the 2D SPGR signal strength equation (e.g., no variations in $B_{1}$ or slice profile shape). Under this assumption, [C](t) could be estimated accurately using only the $T_{1 \text { pre }}$ measurement $(4,7,13)$. This alternative analysis method is known as the "Single- $T_{1 \text { pre }}$ Method" (Method 2 in Ref. 7), in contrast to the Bookend Method, which utilizes both $T_{1 \text { pre }}$ and $T_{1 \text { post }}$.

The $T_{1 \text { pre }}$ and $T_{1 \text { post }}$ measurements were obtained with an ASR pulse sequence. For the acquisition of each line of $k$-space, a nonselective sin/cos adiabatic saturation pulse was applied, followed by a recovery time $\tau$, followed by readout pulses and data acquisition for all slices (17). An adiabatic saturation pulse was used in order to make the $T_{1}$ measurement less sensitive to $B_{1}$ inhomogeneities. The nonselective sin/cos pulse was applied for $102 \mathrm{~ms}$. This pulse started $233 \mathrm{~Hz}$ off-resonance and ended with a maximum $B_{1}$ amplitude of $\sim 0.073$ Gauss. The pulse sequence had conventional, sequential phase encode ordering and interleaved slice ordering. Thus, after the recovery time $\tau$, slices $1,3,5$, then 7 were read out, followed immediately by slices $2,4,6$, then 8 . The pulse sequence was repeated with the same $\tau$ until an entire image had been acquired. One image was acquired for each of four values of $\tau$ as described above.

The peak specific absorption rate (SAR) generated in the breast by the ASR pulse sequence was calculated to be under $8 \mathrm{~W} / \mathrm{kg}$, which is the limit recommended by the 


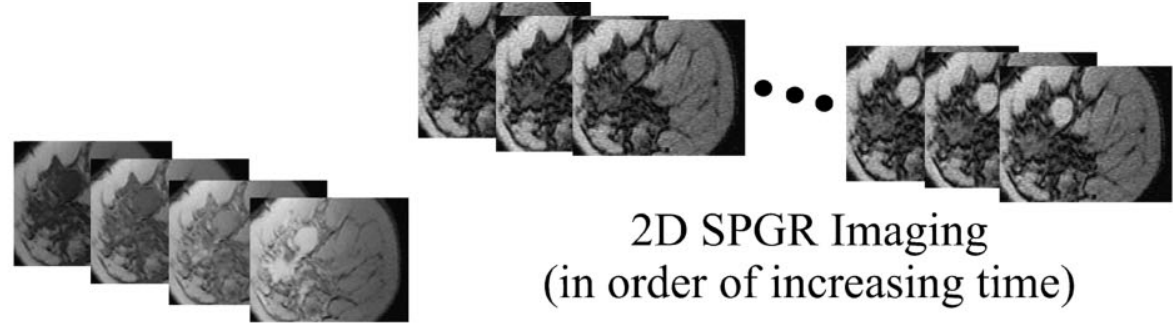

Bookend \#1: ASR Measurement of $\mathrm{T}_{1 \text { pre }}$ (in order of increasing $\tau$ )

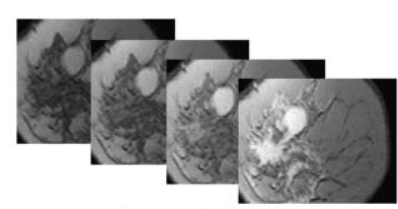

Bookend \#2: ASR Measurement of $\mathrm{T}_{1 \text { post }}$ (in order of increasing $\tau$ )

FIG. 1. Representative ASR and 2D SPGR images (cropped) for a single slice of one patient who had a fibroadenoma (round mass near the top of the images).

United States Food and Drug Administration for a local transmitter coil. This calculation was done using manufacturer-supplied information and was based on the assumption that the transmit/receive breast coil and the transmit/receive head coil behave similarly in terms of RF heating.

Numerical simulations were performed in order to determine the theoretical accuracy of this ASR $T_{1}$ measurement technique. $T_{1}$ was fit to simulated, noiseless, recovery data for $\tau$ values of 230, 470, 877, and $3000 \mathrm{~ms}$. Recovery data were generated for all combinations of the following parameters: $T_{1}$ (400, $\left.1200 \mathrm{~ms}\right) ; T_{2}$ (50, $300 \mathrm{~ms}$ ); $B_{1}$ inhomogeneity (i.e., the actual $B_{1}$ divided by the expected $\left.B_{1}\right)(0.5,0.75,1.0,1.25,2.0)$; and resonant offset $(-25,0,25 \mathrm{~Hz})$. For each case of $T_{1}, T_{2}, B_{1}$ inhomogeneity, and resonant offset, the effect of the adiabatic pulse was calculated by solving the Bloch equations numerically. The readout pulses were assumed to be single-lobed sinc pulses which are typically used in 2D SPGR imaging.

Phantom experiments were also performed to test the accuracy of the ASR technique: $12 \mathrm{~mm}$ - and $5 \mathrm{~mm}$-wide plastic tube phantoms were filled with different aqueous concentrations of $\mathrm{Gd}$ contrast agent providing $T_{1}$ ranging from $1290 \pm 10 \mathrm{~ms}$ down to $450 \pm 5 \mathrm{~ms}$ as measured by inversion recovery (IR). This range of $T_{1}$ was used because it is approximately the range expected in vivo. The IR measurements were performed with a single-slice IR technique with 12 inversion time (TI) values ranging from 50-8000 ms, and TR $=\mathrm{TI}+9000 \mathrm{~ms}$. For this work, the IR measurements were considered the "Gold Standard" measurements of $T_{1}$.

For ASR imaging, the tubes were placed upright at 8 to 9 different positions in a 9-cm high plastic container of water. The transmit/receive breast coil was used to obtain coronal images of this container with a slice thickness of $5 \mathrm{~mm}, 8$ contiguous slices, matrix $=256 \times 128$, and field of view $=16 \mathrm{~cm} . T_{1}$ measurements with ASR were repeated six times for different locations in the water container and different arrangements of the phantom tubes. A total of 816 ASR $T_{1}$ measurements were performed, covering a volume of $830 \mathrm{~cm}^{3}$, which is nearly all the useful imaging volume of the coil. $\tau$ values of $230,635,1329$, and
2920 ms were used to measure the $T_{1}$ of the tubes with $T_{1}>650 \mathrm{~ms}$, while $\tau$ values of $230,432,775$, and $2920 \mathrm{~ms}$ were used to measure the $T_{1}$ of the tubes with $T_{1}<650 \mathrm{~ms}$. These sampling schemes were the same as those which were used for the patients to measure $T_{1 \text { pre }}$ (which was expected to be $>650 \mathrm{~ms}$ ) and $T_{1 \text { post }}$ (which was expected to be $<650 \mathrm{~ms}$ ).

\section{RESULTS}

For the simulations, the ASR technique maintained reasonably small errors $(<10 \%)$ even down to $B_{1}$ inhomogeneities of 0.5 . In phantoms, the ASR technique measured $T_{1}$ accurately (5\%) for all experiments. Figure 1 shows representative ASR and 2D SPGR images. Figure 2 shows the correction that had to be applied to the 2D SPGR signal strength equation for the Bookend Method, as a function of $1 / T_{1}$ for all 14 patients. The ordinate may also be described as the ratio of the actual 2D SPGR signal strength to the

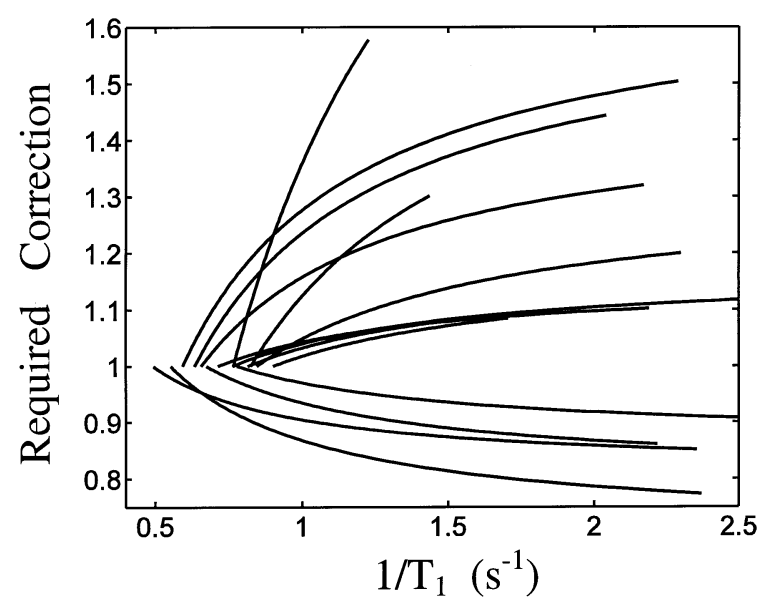

FIG. 2. The correction that had to be applied to the 2D SPGR signal strength equation for the Bookend Method, as a function of $1 / T_{1}$ for all 14 patients. A value greater than 1 indicates that the measured signal was greater than that predicted by the theoretical 2D SPGR signal strength equation. 
FIG. 3. The concentration of Gd in tissue as a function of time $([\mathrm{C}](\mathrm{t}))$ for two different lesions (benign proliferative dysplasia and infiltrating moderately differentiated ductal carcinoma). The solid and dotted lines show the curve fits for the pharmacokinetic model. a: $[\mathrm{C}](\mathrm{t})$ calculated with the Single- $T_{1 \text { pre }}$ Method. For this data, the EF of the benign lesion was 44.4 , compared to 26.8 for the cancerous lesion. b: $[C](t)$ calculated with the Bookend Method. This time, the EF of the cancerous lesion was much higher than that of the benign lesion ( $E F=43.1$ compared to 9.8).

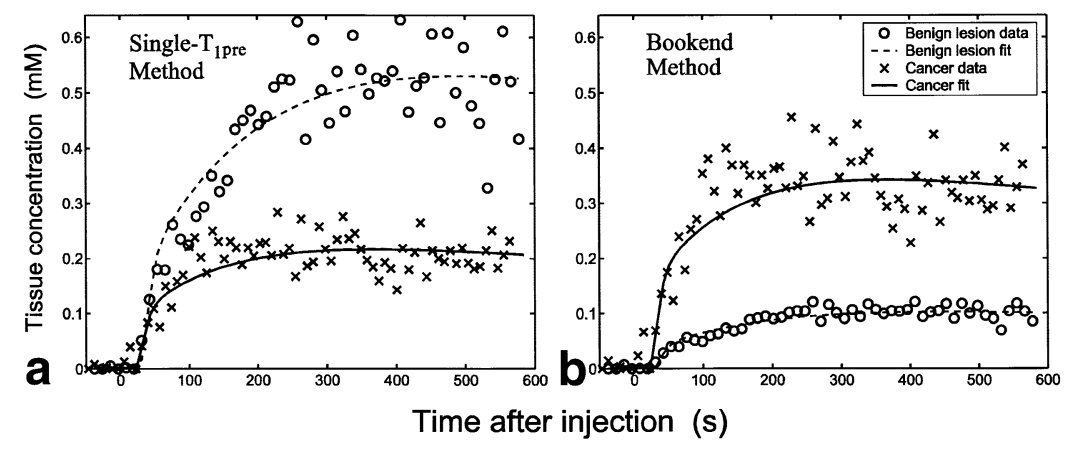

theoretical value predicted by the signal strength equation. Although this ratio is linear with $T_{1}$ (7), the plot was displayed as a function of $1 / T_{1}$ to give a sense of how it can vary wildly from patient to patient with increasing Gd concentration. This figure shows that the actual 2D SPGR signal strength may differ from the theoretical value by factors ranging from $\sim 0.8-1.5$ and underlines the value of the second $T_{1}$ bookend measurement $\left(T_{1 \text { post }}\right)$.

Figure 3 shows example tissue [C](t) data for two lesions from two different patients. One of the lesions was benign (proliferative dysplasia) and the other was cancerous (infiltrating moderately differentiated ductal carcinoma). Both lesions were $\sim 5 \mathrm{~mm}$ in size. Figure 3a shows [C](t) for the lesions as calculated by the Single- $T_{1 \text { pre }}$ method, whereas Fig. 3b shows [C](t) as calculated by the Bookend Method. Figure 3a gives the false impression that the benign lesion absorbed a higher concentration of Gd than the cancerous lesion. In fact, as shown in Fig. 3b, the cancerous lesion had a much more aggressive enhancement than the benign lesion.

Figure 4 shows EF values for all the lesions for (4a) the Single- $T_{1 \text { pre }}$ Method and (4b) the Bookend Method. For each of the two methods, an EF threshold between cancerous and benign lesions was chosen which would maximize sensitivity and specificity. For the Single- $T_{1 \text { pre }}$ Method, the sensitivity and specificity were $80 \%$ and

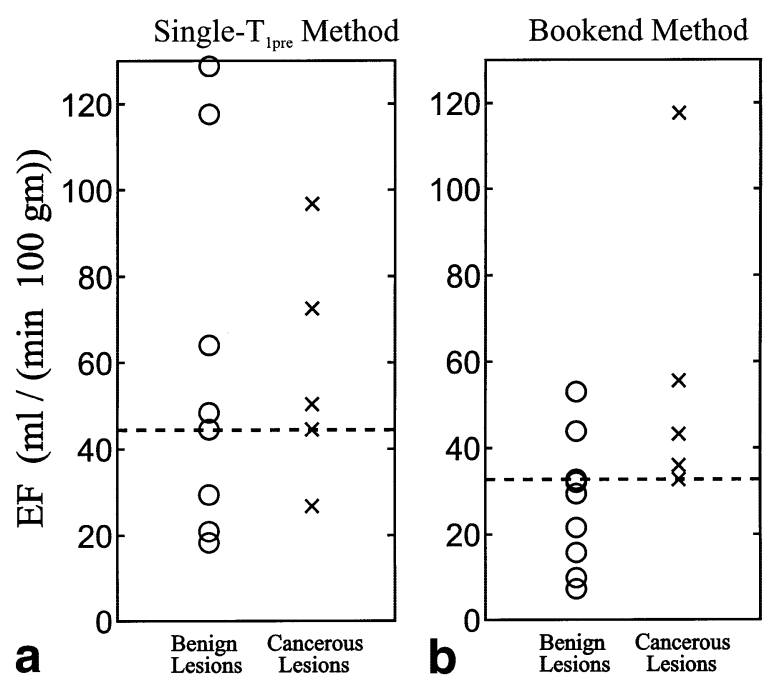

FIG. 4. EF vs. lesion type for the Single- $T_{1 \text { pre }}$ Method (a) and the Bookend Method (b). The EF thresholds chosen were (a) 44.4 and (b) 32.7
$56 \%$, respectively. For the Bookend Method, the sensitivity and specificity were $100 \%$ and $78 \%$, respectively.

For the Bookend Method analysis, the average value of $v$ was found to be $0.59 \pm 0.31$ for malignant lesions and $0.70 \pm 0.26$ for benign lesions. The average uncertainty in the curve fits for $\mathrm{EF}$ was $7 \%$ (range 1-10\%), whereas that for $v$ was $6 \%$ (range 1-15\%). The average measured value of $T_{1 \text { pre }}$ was $1256 \pm 142 \mathrm{~ms}$ for the cancerous lesions and $1537 \pm 265 \mathrm{~ms}$ for the benign lesions. For $T_{1 \text { post }}$, these numbers were $446 \pm 151 \mathrm{~ms}$ and $508 \pm 148 \mathrm{~ms}$, respectively.

\section{DISCUSSION}

The results of this study show that a single preinjection $T_{1}$ measurement is not sufficient for estimating [C](t) reliably from rapid 2D SPGR imaging for pharmacokinetic modeling. This is because the discrepancy between the measured 2D SPGR signal strength and its theoretical value is not consistent from lesion to lesion (Fig. 2). This inconsistency is presumably due to unexpected variations in the $B_{1}$ or slice-select profile, which could be caused by factors such as patient-to-patient variations in breast geometry or inconsistently set transmit gains. The Bookend Method, however, circumvents these problems by obtaining a caseby-case empirical calibration of 2D SPGR signal strength vs. $T_{1}$. The $[\mathrm{C}](\mathrm{t})$ as estimated by the Bookend Method should therefore be much more accurate than $[\mathrm{C}](\mathrm{t})$ estimated by the Single- $T_{1 \text { pre }}$ Method. This is consistent with the fact that the Bookend Method provided higher sensitivity and specificity for this study than the Single- $T_{1 \text { pre }}$ Method, given the assumption that there should be a significant separation in EF values between cancerous and benign lesions.

For the Bookend Method, it is important that the accuracy of the $T_{1 \text { pre }}$ and $T_{1 \text { post }}$ measurements not be adversely affected by the same variations in the $B_{1}$ or slice-select profile which render the Single- $T_{1 \text { pre }}$ Method unreliable. For this reason, we chose a $T_{1}$ measurement method (ASR) which would be relatively unaffected by $B_{1}$ inhomogeneities. The ASR method demonstrated low systematic errors $(<5 \%)$ for both the simulations and phantom experiments.

The principal drawback of the Bookend Method is that the exam time must be lengthened to accommodate the $T_{1 \text { post }}$ measurement. If limiting the total exam time is crucial, both bookend $T_{1}$ measurements could be performed with a faster, more efficient method such as one of the Look-Locker techniques (18). Whatever fast $T_{1}$ measurement method is chosen, it must maintain the same spatial resolution as the $2 \mathrm{D}$ 
SPGR imaging and not suffer problems from $B_{1}$ inhomogeneities or multislice interference.

One of the weaknesses of this study, and most other studies which have been published on breast MR, is the lack of a true arterial input function (19). The assumed input function used was based on the approximate average of some input function data obtained from a small group of subjects $(15,16)$. However, changes in the input function from one individual to another could cause significant changes in the $[\mathrm{C}](\mathrm{t})$ data, leading to unwanted variations in the measured pharmacokinetic parameters (19). Based on the work by Port et al. (fig. 7 of Ref. 19), it can be estimated that using the same input function for all patients will cause unwanted errors in the pharmacokinetic parameters of about $25 \%$ on average. However, a certain fraction of patients $(\sim 15 \%$ in Port et al.) will have input functions which deviate very significantly from the average, leading to very large ( $>40 \%$ ) errors. Therefore, there is a need to develop rapid, accurate methods for measuring the arterial input function while simultaneously acquiring the Gd concentration-vs.-time in the breast tissue ([C](t)).

\section{CONCLUSIONS}

For dynamic contrast-enhanced MR imaging of the breast, when estimating $[\mathrm{C}](\mathrm{t})$ for a lesion from $T_{1}$-weighted $2 \mathrm{D}$ SPGR imaging, it is not sufficient to use a single preinjection $T_{1}$ measurement ( $T_{1 \text { pre }}$ ) to calibrate the signal strength equations used to convert signal strength to $T_{1}$. This is because the discrepancy between the measured signal strength and its theoretical value is not consistent from lesion to lesion. A second $T_{1}$ measurement, performed after the dynamic contrast-enhanced imaging $\left(T_{1 \text { post }}\right)$, must be done to obtain a case-by-case empirical calibration of signal strength vs. $T_{1}$. This improves the accuracy of the estimated $[\mathrm{C}](\mathrm{t})$ and ultimately improves sensitivity and specificity for the pharmacokinetic parameter(s) used to differentiate benign from malignant tumors (e.g., the extraction-flow product which was used in this work). For this particular patient study $(n=14)$, using the two $T_{1}$ measurements (the "Bookend Method"), gave a sensitivity and specificity of $100 \%$ and $78 \%$, respectively, compared to use of the preinjection $T_{1}$ measurement alone, which had a poorer performance ( $80 \%$ and $56 \%$, respectively). The power of the Bookend Method is that, as long as $T_{1 p r e}$ and $T_{1 \text { post }}$ are measured accurately, [C](t) can be estimated reliably from $2 \mathrm{D}$ SPGR signal strengths without any a priori knowledge of system imperfections.

\section{ACKNOWLEDGMENTS}

The authors thank the Children's Hospital of Eastern Ontario and the Kingston General Hospital for assistance and cooperation with this research. We also thank Peggy Florack and Lisa Johnston of the Women's Breast Health Centre of the Ottawa Hospital for assistance with patient recruitment and follow-up, and Rick L'Heroux for clinical assistance with the patient studies performed in Ottawa. We thank Dr. Brian Pearse for help with the patient studies in Kingston, and Dr. Albert Cross for help with maintenance of the breast RF coil, and Dr. Julia Wallace, Cyndi Little, Dr. Peter Shragge, and Jennifer Mallalieu for help with the Adiabatic Saturation Recovery work.

\section{REFERENCES}

1. Padhani AR. Dynamic contrast-enhanced MRI in clinical oncology: current status and future directions. J Magn Reson Imag 2002;16:407422.

2. Warner E, Plewes DB, Shumak RS, Catzavelos GC, Di Prospero LS, Yaffe MJ, Goel V, Ramsay E, Chart PL, Cole DEC, Taylor GA, Cutrara M, Samuels TH, Murphy JP, Murphy JM, Narod SA. Comparison of breast magnetic resonance imaging, mammography, and ultrasound for surveillance of women at high risk for hereditary breast cancer. J Clin Oncol 2001;19:3524-3531.

3. Brown J, Buckley D, Coulthard A, Dixon AK, Dixon JM, Easton DF, Eeles RA, Evans DGR, Gilbert FG, Graves M, Hayes C, Jenkins JPR, Jones AP, Keevil SF, Leach MO, Liney GP, Moss SM, Padhani AR, Parker GJM, Pointon LJ, Ponder BAJ, Redpath TW, Sloane JP, Turnbull LW, Walker LG, Warren RML. Magnetic resonance imaging screening in women at genetic risk of breast cancer: imaging and analysis protocol for the UK Multicentre Study. Magn Reson Imag 2000;18:765-776.

4. Hulka CA, Smith BL, Sgroi DC, Tan L, Edmister WB, Semple JP, Campbell T, Kopans DB, Brady TJ, Weisskoff RM. Benign and malignant breast lesions: differentiation with echo-planar MR imaging. Radiology 1995;197:33-38.

5. Hulka CA, Edmister WB, Smith BL, Tan L, Sgroi DC, Campbell T, Kopans DB, Weisskoff RM. Dynamic echo-planar imaging of the breast: experience in diagnosing breast carcinoma and correlation with tumor angiogenesis. Radiology 1997;205:837-842.

6. Brookes JA, Redpath TW, Gilbert FJ, Needham G, Murray AD. Measurement of spin-lattice relaxation times with FLASH for dynamic MRI of the breast. Br J Radiol 1996;69:206-214.

7. Cron GO, Santyr G, Kelcz F. Accurate and rapid quantitative dynamic contrast-enhanced breast MR imaging using spoiled gradient-recalled echoes and bookend $T_{1}$ measurements. Magn Reson Med 1999;42:746-753.

8. Tofts PS, Brix G, Buckley DL, Evelhoch JL, Henderson E, Knopp MV, Larsson HBW, Lee TY, Mayr NA, Parker GJM, Port RE, Taylor J, Weisskoff RM. Estimating kinetic parameters from dynamic contrast-enhanced $T_{1}$-weighted MRI of a diffusable tracer: standardized quantities and symbols. J Magn Reson Imag 1999;10:223-232.

9. Parker GJM, Suckling J, Tanner SF, Padhani AR, Revell PB, Husband JE, Leach MO. Probing tumor microvascularity by measurement, analysis and display of contrast agent uptake kinetics. J Magn Reson Imag 1997; $7: 564-574$.

10. Rijpkema M, Kaanders JHAM, Joosten FBM, van der Kogel AJ, Heerschap A. Method for quantitative mapping of dynamic MRI contrast agent uptake in human tumors. J Magn Reson Imag 2001;14:457-463.

11. Henderson E, Rutt BK, Lee TY. Temporal sampling requirements for the tracer kinetics modeling of breast disease. Magn Reson Imag 1998; 16:1057-1074.

12. Donahue K, Burstein D, Manning W, Gray M. Studies of gadopentetate dimeglumine relaxivity and proton exchange rates in tissue. Magn Reson Med 1994;32:66-76.

13. Tofts PS, Berkowitz B, Schnall MD. Quantitative analysis of dynamic Gd-DTPA enhancement in breast tumors using a permeability model. Magn Reson Med 1995;33:564-568.

14. Donahue KM, Weisskoff RM, Burstein D. Water diffusion and exchange as they influence contrast enhancement. J Magn Reson Imag 1997;7: 102-110.

15. Fritz-Hansen T, Rostrup E, Larsson HBW, Søndergaard L, Ring P, Henriksen O. Measurement of the arterial concentration of Gd-DTPA using MRI: a step toward quantitative perfusion imaging. Magn Reson Med 1996;36:225-231.

16. Weinmann HJ, Laniado M, Mutzel W. Pharmacokinetics of GdDTPA dimeglumine after intravenous injection into healthy volunteers. Physiol Chem Phys Med NMR 1984;16:167-172.

17. Bendall MR, Pegg DT. Uniform sample excitation with surface coils for in vivo spectroscopy by adiabatic rapid half passage. J Magn Reson 1986;67:376-381.

18. Hendersen E, McKinnon G, Lee TY, Rutt B. A fast 3D Look-Locker method for volumetric $T_{1}$ mapping. Magn Reson Imag 1999;17:1163-1171.

19. Port RE, Knopp MV, Brix G. Dynamic contrast-enhanced MRI using Gd-DTPA: interindividual variability of the arterial input function and consequences for the assessment of kinetics in tumors. Magn Reson Med 2001;45:1030-1038.

20. Kelcz F, Santyr GE, Cron GO, Mongin SJ. Application of a quantitative model to differentiate benign from malignant breast lesions detected by Gd-enhanced MRI. J Magn Reson Imag 1996;6:743-752. 